\title{
Effectiveness of the Wavelet Transform on the Surface EMG to Understand the Muscle Fatigue During Walk
}

\author{
M. S. Hussain ${ }^{1}$, Md. Mamun ${ }^{2}$ \\ ${ }^{1}$ School of Electrical and Information Engineering, University of Sydney, NSW 2006, Australia \\ ${ }^{2}$ Smart Engineering System Research Group, Universiti Kebangsaan Malaysia, 43600, Bangi, Selangor, Malaysia, \\ e-mail: mdmamun.ukm@gmail.com
}

\begin{abstract}
Muscle fatigue is the decline in ability of a muscle to create force. Electromyography (EMG) is a medical technique for measuring muscle response to nervous stimulation. During a sustained muscle contraction, the power spectrum of the EMG shifts towards lower frequencies. These effects are due to muscle fatigue. Muscle fatigue is often a result of unhealthy work practice. In this research, the effectiveness of the wavelet transform applied to the surface EMG (SEMG) signal as a means of understanding muscle fatigue during walk is presented. Power spectrum and bispectrum analysis on the EMG signal getting from right rectus femoris muscle is executed utilizing various wavelet functions (WFs). It is possible to recognize muscle fatigue appreciably with the proper choice of the WF. The outcome proves that the most momentous changes in the EMG power spectrum are symbolized by WF Daubechies45. Moreover, this research has compared bispectrum properties to the other WFs. To determine muscle fatigue during gait, Daubechies45 is used in this research to analyze the SEMG signal.
\end{abstract}

Keywords: Electromyography, surface electromyography, wavelet, bispectrum

\section{INTRODUCTION}

$\mathrm{M}$ USCLE FATIGUE is a condition when the ability of the muscle to contract and produced force is reduced. Unlike subjective typical evaluations, which typically define the point in time when the subject can no longer perform tasks, EMG signal analysis can provide a continuous measurement of a muscle's metabolic state throughout a sustained fatiguing contraction [1]. However, muscle fatigue threshold cannot be defined as a simple function of muscle load magnitude and timing because muscle characteristics and capabilities vary from person to person.

The electrical activity of muscles is represented by Electromyography (EMG) signal. A muscle is composed of many Motor Units (MUs). An EMG signal is realized instantly from the muscle or from the skin using surface electrodes. Understandingly, it demonstrates noise as well as Motor Unit Action Potentials (MUAP). While increasing muscle force, the number of MUAP employed at rising firing rates is enlarged. The raw EMG signal depicts the increment in the Interference Pattern (IP) [2]. The firing pulses are normally considered a random function of time, which is non-Gaussian in nature [3]. Quantitative analysis of the IP is useful in the diagnosis of neuromuscular disorders.

Undetected muscle fatigue can cause injury. Within static sub maximal contractions both amplitude and frequency based analysis parameters show time domain changes due to muscle fatigue. Progressive muscle fatigue can be tracked by monitoring the changes in the characteristic frequencies of the EMG spectrum. There are a number of techniques that can be used to determine the level of fatigue in a subject. Due to recruitment of motor units, the amplitude shows an increase, whereas the frequency based mean or median frequency of the total power spectrum show a decrease over contraction time $[4,5]$. The regression coefficient of the median or mean frequency slope towards lower frequencies can be used as a non-invasive fatigue index for the investigated muscle.

To analyze the frequency components of the EMG signal, the mean and median frequencies are the most important parameters. Lindstrom et al. [6] have developed a general mathematical model of the EMG power spectrum density (PSD) and have shown that both the amplitude increase and shifts to lower frequencies can be explained by conduction velocity changes during sustained contraction. Stulen and DeLuca [7] have shown that the characteristic frequencies of the PSD such as mean and median frequencies are linearly proportional to the conduction velocity. Using spectral analysis, Stulen and DeLuca observed a shift in the EMG frequency towards lower frequencies. They demonstrated that the median frequency parameters of the power spectrum provided a reliable consistent estimate of the frequency shift.

In the past years, several computer-aided techniques for IP analysis have been proposed including the following: turns amplitude analysis [8], decomposition methods [7], and power spectrum analysis [9]. Bispectrum analysis, a particular form of higher-order spectra (HOS), was used by Kaplanis and Pattichis [3] for analyzing the "Biceps Brachii" muscle. The analysis revealed that SEMG turn into less Gaussian on escalating mean voluntary contraction (MVC). Different research works using HOS showed that MUAP waveform increased according to the increase in load weight where there is no involvement of Motor Units (MUs) recruitment in the resting muscle [10-12]. The point at which a contraction can no longer be maintained (the failure point) has been generally designated as the point at which the muscle is said to fatigue [13]. This information can also be very useful for identifying muscle fatigue while analyzing the SEMG signal.

In this research, various WFs are employed to decompose SEMG using Discrete Wavelet Transform (DWT). Median 
frequency is considered for the EMG power spectrum analysis during an 8-trial walk (slow to fast). Consecutively, to take out new parameters that could develop the diagnostic character of SEMG, HOS in SEMG signal analysis is used in this research. To analyze SEMG signals and to detect muscle fatigue during a 3-trial (slow, medium and fast) walking process, Bispectrum analysis is introduced. In this study, WFs Haar, Daubechies and Symmlet are used for the WT.

Results suggest that a specific WF provides the best contrast for the analysis of SEMG signal to identify muscle fatigue. According to this research, db45 (Daubechies WF) present the best contrast while analyzing the EMG signal using both power spectrum and bispectrum. Db45 has been used as the main WF to identify muscle fatigue during gait. During the study, it was also demonstrated that median frequency amplifies with the enlargement of muscle contraction. The increase of muscle contraction also makes the signal less Gaussian. Furthermore, we have also noticed that the Linearity test follows the reverse pattern with the measure of Gaussianity.

\section{WAVELET DECOMPOSITION}

The DWT is computed by successive low-pass and highpass filtering in the discrete-time domain. The DWT of a signal $x[n]$ is calculated by passing it through a series of filters. First, the samples $x[n]$ are passed through a low-pass filter with impulse response $\mathrm{g}[\mathrm{n}]$, resulting in a convolution $y[n]$ of the two. The signal also goes simultaneously through a high-pass filter with impulse response $h[n]$. The outputs give the detail coefficients (from the high-pass filter, $y_{\text {high }}[n]$ ) and the approximation coefficients (from the lowpass filter, $\left.y_{\text {low }}[n]\right)$. The filter outputs are then down sampled (or sub sampled) as given by

$$
\begin{aligned}
& y_{\text {low }}[n]=\sum_{k=-\infty}^{\infty} x[k] \cdot g[2 n-k] \\
& y_{\text {high }}[n]=\sum_{k=-\infty}^{\infty} x[k] \cdot h[2 n-k]
\end{aligned}
$$

The aptitude of DWT to extract features from the signal is reliant on the suitable option of the mother wavelet function. Some of the popular standard families of the wavelet basic functions are Haar, Daubechies, Coeiflet, Symmlet, Morlet and Mexican Hat. Even though there is no well-defined rule for selecting a wavelet basis function in a particular application or analysis, some properties of the wavelets make a specific mother wavelet more suitable for a given application and signal type [14].

There are some general guidelines to select the wavelets, such as Db4 is more suitable for signals that have "linear approximation" over the support of four samples, while Db6 is better suited for a signal approximated by a quadratic function over the support of six, coiflet 6 provides better data compression results while Db4 is more suitable for feature extraction [15]. The properties of wavelet functions used in this paper are shown in Table1.
Table 1. Properties of Wavelet Function

\begin{tabular}{|l|l|l|l|}
\hline & Haar & DbN & SymN \\
\hline Orthogonal & Yes & Yes & Yes \\
\hline Time Support & {$[0,1]$} & {$[0,2 \mathrm{~N}-1]$} & {$[0,2 \mathrm{~N}-1]$} \\
\hline Frequency Support & $1 / \omega$ & $1 / \omega^{0.2 \mathrm{~N}}$ & $1 / \omega^{0.2 \mathrm{~N}}$ \\
\hline Regularity & 0 & $0.2 \mathrm{~N}$ & $0.2 \mathrm{~N}$ \\
\hline Symmetry & Yes & No & Yes \\
\hline Zero Moment & 1 & $\mathrm{~N}$ & $\mathrm{~N}$ \\
\hline
\end{tabular}

\section{BISPECTRUM ESTIMATION PROCESS}

The bispectrum is a particular form of HOS. As it is the simplest to compute among other HOS forms, it is the most accessible. The two-dimensional discrete-time Fourier transform of the third order cumulant gives the bispectrum.

Knowing the frequency components, $X(k)$, of the output signal $x(k)$, the bispectrum, $B_{x}(k, l)$, can be estimated using equation (4).

$$
B_{x}(k, l)=E\left\{X(k) X(l) X^{*}(k+1)\right\}
$$

where $E\{$.$\} denotes the statistical expectation, k, l$ are the discrete frequency components and $*$ denotes the complex conjugate.

Given a set of real observations $\{x(n)\}$ for $n=0,1,2, \ldots$, $\mathrm{N}-1$, it is assumed that the data set is stationary. The bispectrum estimation algorithm is given as follows [16].

1. Subdivide the data into $G$ segments of $M$ samples, where each segment $(N=G \times M)$. The new denotation of $\{x(n)\}$ is $x g(m)$ where $m=0,1,2, \ldots ., M-1$ and $g=$ $0,1,2, \ldots, G-1$. The sample size $(M)$ is chosen as the number of frequency points of the FFT (Fast Fourier Transform) for better evaluation.

2. From each record of any segment, subtract the average (mean) value. To achieve a length $M$ for the FFT, add zeroes to each division such that $M$ is a power of 2 .

3. Multiply each section by an appropriate data window to manage the consequence of spectral leakage. A onedimensional Hamming or Hanning window can be used.

4. Inside each segment, calculate the DFT (Discrete Fourier Transform) coefficients.

5. For the principal domain, compute the probable bispectrum by using DFT coefficients.

The bispectrum estimation of the given data is averaged over the ' $G$ ' pieces. The power spectrum can also be expected during the process.

\section{GAUSSIANITY AND LINEARITY}

To quantify the non-Gaussianity of a random process, the normalized bispectrum gives the bicoherence $B \_n(k, l)$ which is estimated by

$$
B \_n(k, l)=B(k, l) / \sqrt{P(k) P(l) P(k+l)}
$$

where $B(k, l)$ is the bispectrum and $P($.$) is the power$ spectrum. Bicoherence is a mixed function of second- and third-order statistics that is the power spectrum and bispectrum. The third-order and fourth-order cumulant gives the skewness. The skewness function of a linearly filtered 
non-Gaussian signal is flat but the skewness function of the output signal of a non-linear system is not flat [17]. If a signal is Gaussian then its bispectrum is zero. The theoretical bispectrum of any Gaussian signal is identically zero. Researchers reported that the bispectrum is zero for any independent and identically distributed signal with a symmetric probability density function (pdf) whereas skewed signals have an asymmetric pdf whose bispectrum is non-zero $[18,19]$. It is also reported that any signal which is not white has a non-zero bispectrum. The power spectrum and bispectrum of a non-Gaussian random signal always carry a constant value.

The test of Gaussianity $S_{g}$ is based on the mean bicoherence power defined as the summation performed over the non-redundant region, which is given as

$$
S_{g}=\sum B_{n}(k, l)
$$

The Gaussianity test (actually zero-skewness test) involves deciding whether the estimated bicoherence is zero $[17,20]$. The Linearity test involves deciding whether the estimated bicoherence is constant in the bi-frequency domain, employing a measure of the difference $(d R)$ between a theoretical and an estimated interquartile range $R$. The Gaussianity test is performed to understand muscle contraction to identify fatigue.

\section{Methodology}

Eleven (11) separate EMG data files are used in this research. For the simulation of the algorithm, Raw EMG signals are collected from Motion Lab Systems, Inc. [21]. SEMG is documented from the right "rectus femoris" muscle which is taken from a normal subject (teenage male). Overall, 800 samples per second are recorded for all analog channels. DWT with five different WFs (haar, db2, db45 sym4 and sym5) is used to decompose SEMG signals. Once the WT is done, the wavelet coefficients are used for power spectrum and bispectrum analysis.

FFT techniques are used to attain the power, where Hanning window is used with a 128 point FFT. The median frequency is considered by finding the middle frequency value of the power spectrum. For the bispectrum analysis, the signal is segmented at 256 points and 0.51 smoothing. The $\mathrm{N}$ numbers of wavelet coefficients are used for the bispectrum estimation. The $\mathrm{N}$ data are divided into $\mathrm{G}$ section of 256 samples. Sample size 256 is the number of frequency points for the FFT. From every record of that segment, the average value of each segment is deducted. An appropriate data window is multiplied with each segment to manage the effect of spectral leakage. A one-dimensional Hanning is used for this case. Within each segment, the DFT coefficient is computed. Estimated bispectrum is computed by using DFT coefficients for the principle domain. Next, the bispectrum estimate for the given data is averaged over $G$ pieces. Finally, the Gaussianity and Linearity tests are executed. The DWT is executed using MATLAB Wavelet toolbox and the power spectrum. The bispectrum analysis is also achieved with MATLAB.

Median frequency of the power spectrum is used to calculate the muscle contraction at various walking trials, which is valuable to sense muscle fatigue. Based on the normalized bispectrum (bicoherence), the Gaussianity and Linearity tests are also carried out for the SEMG signals to determine fatigue. The process of power spectrum and bispectrum analysis of SEMG is shown in Fig.1.

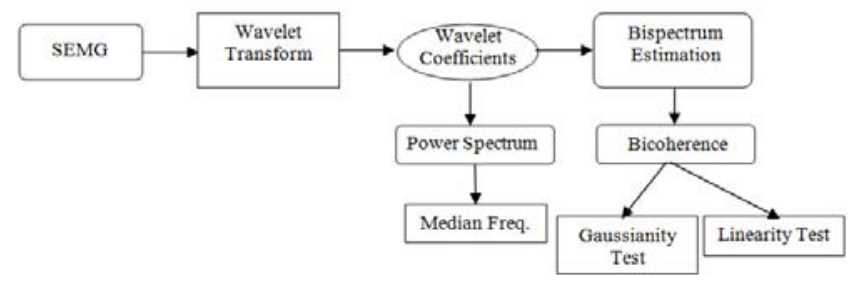

Fig.1. Power Spectrum and Bispectrum Analysis of SEMG

\section{RESUlts}

Fig. 2 shows the median frequency of the 8-trial walk (slow to fast) using various WFs and Fig. 3 shows the Gaussianity test of the 8-trial walk (slow to fast) using various WFs, where each trial walk lasted 15 minutes. Fig. 4 gives the median frequency for the 3-trial walk (slow, medium and fast, where 1 represent slow, 2 represent medium and 3 represent fast in the $x$ axis) to determine muscle fatigue and Fig.5 shows the Gaussianity test for the 3-trial walk (slow, medium and fast, where 1 represent slow, 2 represent medium and 3 represent fast in the $x$ axis) to identify muscle fatigue. Fig.6 shows the Linearity test for a 3-trial walk (slow, medium and fast, where 1 represent slow, 2 represent medium and 3 represent fast in the $x$ axis) showing reverse pattern of the Gaussianity test. Median frequency and Gaussianity test of the muscle rest position and maximum contraction position are also represented in Fig.7 and Fig.8 to verify the performance of WF db45. It is observed that:

1. Compared to the other WFs, WF db45 presents the best contrast in median frequencies as shown in Fig. 2.

2. To make the SEMG signal less Gaussian and linear, WF is compared to other WFs. The best contrast in the bispectrum analysis is represented by $\mathrm{db} 45$, which is exposed in Fig.3.

3. Muscle fatigue is significantly identified when using WF db45 during power spectrum analysis as it is very clear that during the fast walking trial the spectrum is low (Fig.4).

4. Gaussianity test using db45 shows that the Gaussianity increased from trial 1 (slow walking) to trial 3 (fast walking) indicating decrease of muscle contraction during the trial (Fig.5). The achieved results support the outcome of Kaplanis et al. [3], who have also used Bispectrum analysis for analyzing the biceps brachii muscle, where SEMG becomes less Gaussian with increased muscle contraction.

5. Linearity test follows the reverse pattern of the Gaussianity test where the signal becomes more linear at trial 2 (medium walking) and less linear at trial 3 (fast walking) (Fig.6).

6. Median frequency amplifies with a raise of muscle contraction as shown in Fig.7.

7. SEMG signal distribution is non-Gaussian at the muscle's maximum contraction stage comparing to its resting stage (Fig.8). 


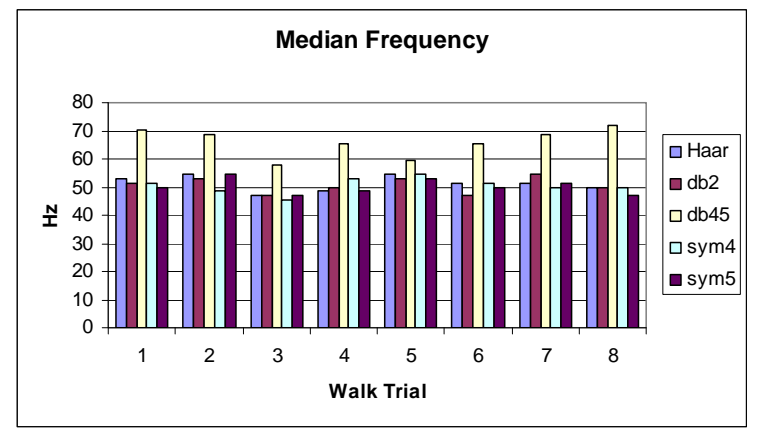

Fig.2. Median frequency of the 8-trial walk using various WFs



Fig.3. Gaussianity test of the 8-trial walk using various WFs

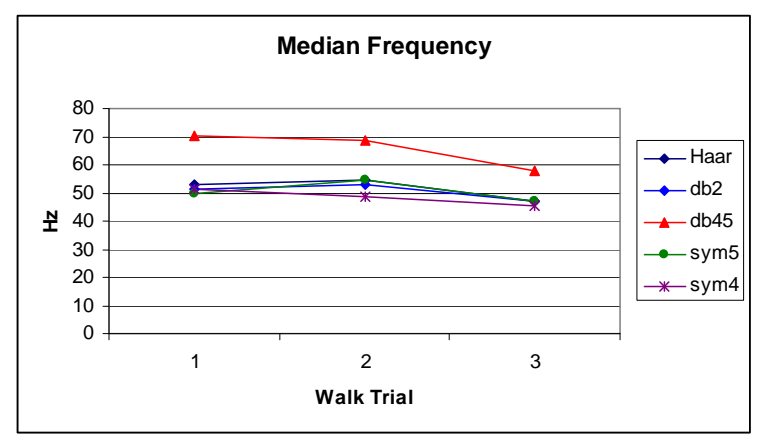

Fig.4. Median frequency for 3-trial to identify muscle fatigue

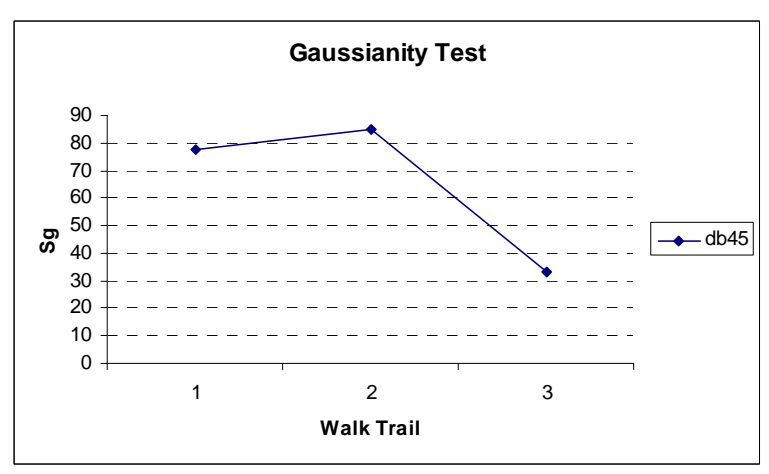

Fig.5. Gaussianity test for the 3-trial walk to identify muscle fatigue

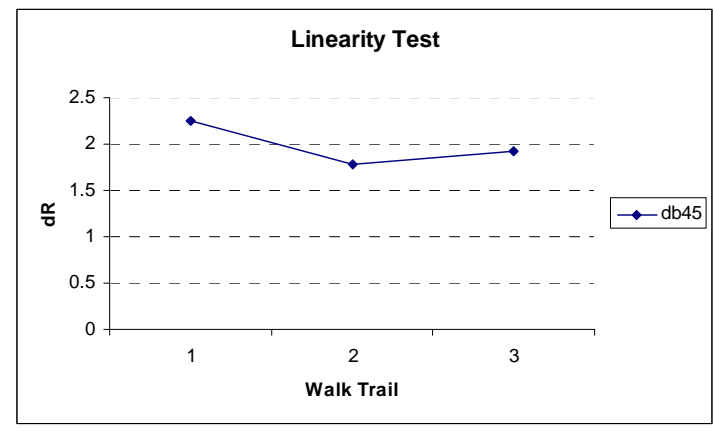

Fig.6. Linearity test for the 3-trial walk showing reverse pattern of the Gaussianity test



Fig.7. Median frequency at the muscle's rest position and maximum contraction position

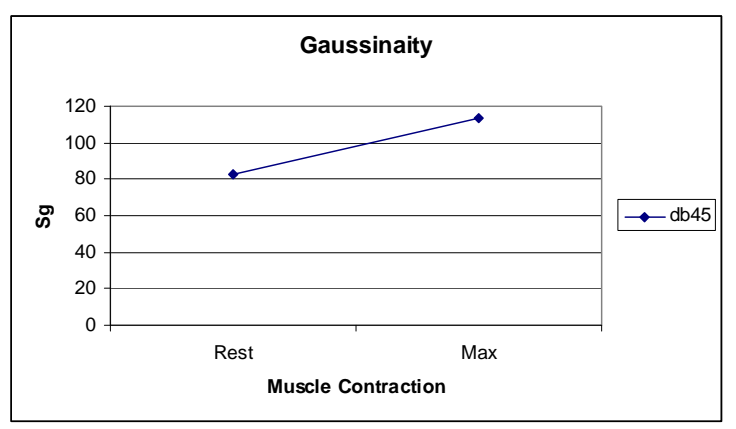

Fig.8. Gaussianity test at the muscle's rest position and maximum contraction position

\section{DISCUSSION}

Median frequency is used to measure the shift to lower frequencies. This shifting is correlated with the changes in the MU and changes in the firing characteristics of the MU [22]. In the past, spectral analysis of the SEMG has demonstrated a significant change of the spectrum of the signal due to muscle fatigue $[6,7]$. However, the change was small because the shape of the spectrum of SEMG varied, so it was difficult to provide an objective and numeric parameter to distinguish between SEMG. Recent research also demonstrates that using WT, the difference between the SEMG corresponding to fatigue muscles and non-fatigue muscles is highlighted when using sym4 and sym5 WFs [14]. 
From the result obtained in this research during the 8-trial walk, it is evident that WF db45 shows the best contrast while analyzing SEMG signal using both power spectrum and bispectrum. WF db45 provides better contrast in the SEMG signal analysis compared to the other WFs, as shown in Fig.2. According to Fig.4, the WF haar, db2, sym4 and sym5 show similar results in $50 \mathrm{~Hz}$ range where there is a shift to lower frequencies in the median frequency from trial 1 (slow walk) to trial 3 (fast walk) indicating muscle fatigue as in $[6,7]$. The WF db45 shows higher median frequency values compared to the other four WFs (haar, db2 sym 4 and sym5) within the range 50 to $70 \mathrm{~Hz}$. The median frequency representing muscle contraction using db45 has significant variations in the signal. The shift to lower median frequency indicates presence of muscle fatigue during the walking trial. In this case, the difference between the SEMG corresponding to fatigue muscle and non-fatigue muscles is best highlighted when using db45 WF, which is better than sym 4 and sym 5 proposed by Kumar et al. [14], which is shown in Fig.2. Gaussianity test as shown in Fig.3 also shows that db45 can show significant variation on the Gaussianity tests making the SEMG signal less Gaussian compared to the other WFs.

Gaussianity test using db45 from Fig.5 shows that the Gaussianity increased from trial 1 (slow) to trial 3 (fast). The increase of Gaussianity during the 3-trial walk determines that there is decrease of muscle contraction indicating muscle fatigue as in [13]. Though there is a rise in $S_{g}$ between trial 1 (slow walk) and trial 3 (fast walk), the fatigue will not necessarily be always linear in nature during walk. However, when there is fatigue, $S_{g}$ will fall, which can be seen clearly in Fig.5. This also concludes that there is a change of MU recruitment during the third walking trial as in $[10,12]$. A measure of Linearity based on whether or not the estimated bicoherence is constant, follows the reverse pattern with that of Gaussianity. Fig.6 shows that the Linearity test follows the reverse pattern of the Gaussianity test where the signal becomes linear at trial 2 (medium walk) and less linear at trial 3 (fast walk).

During this research, a test using the muscle in resting position and maximum contraction position was performed to check the performance of WF db45. Comparison study of Hagberg and Ericson found that mean power frequency was lower at low contraction levels when measured against high contraction levels [23]. Similar results were also accomplished by Moritani et al., where momentous raise in SEMG mean/median frequency was found with increasing force [24]. These research results showed that for maximum contraction level there was considerable raise in the median frequency from the resting state of the muscle. The outcome by using wavelet db45 [23, 24] is shown in Fig.7. This indicates that there are changes in the MU and changes in the firing characteristics of the MU [22]. During the bispectrum analysis, it was also demonstrated that the signal is more Gaussian at rest position and less Gaussian at maximum contraction level as in [3] and shown in Fig.8.

\section{CONCLUSION}

The results of this work demonstrate that by using wavelets, the difference of muscle contractions and fatigue is significantly identified when using wavelet function $\mathrm{db} 45$. Both the power spectrum and bispectrum analysis showed similar results while analyzing muscle fatigue during gait. The study also shows that boosted muscle contraction level (rest to max) leads to an enlargement in median frequencies representing changes in the MUs recruitment. Additionally, augment in the muscle's contraction level (rest to max) makes the SEMG less Gaussian and more linear.

\section{REFERENCES}

[1] Emley, M.S., Gilmore, L.D., Roy, S.H. (1992). Electromyography: Unlocking the secrets of back pain. IEEE Potentials, 11 (2), 25-30.

[2] Broman, H., De Luca, C.J., Mambrito, B. (1985). Motor unit recruitment and firing rates interaction in the control of human muscles. Brain Res., 337, 311-319.

[3] Kaplanis, P.A., Pattichis, C.S., Hadjileontiadis, L.J., Panas S.M. (2000). Bispectral analysis of surface EMG. In 10th Mediterranean Electrotechnical Conference (MELECON 2000), 29-31 May 2000. IEEE, Vol. 2, 770-773.

[4] Edwards, R.H.T. (1981). Human muscle function and fatigue. In Proceeding of the Ciba Foundation Sympozium. Wiley, 1-18.

[5] Phinyomark, A., Limsakul, C., Phukpattaranont, P. (2011). Application of wavelet analysis in EMG feature extraction for pattern classification. Meas. Sci. Rev., 11 (2), 45-52.

[6] Lindstrom, L., Kadefors, R., Petersen, I. (1977). An electromyographic index for localized muscle fatigue. J. Appl. Physiol., 43, 750-754.

[7] Stulen, F.B., De Luca, C.J. (1981). Frequency parameters of myoelectric signal as a measure of muscle conduction velocity. IEEE Trans. Biomed. Eng., 28 (7), 515-523.

[8] Diószeghy, P., Egerházi, A., Molnár, M., Mechler, F. (1996). Turn-amplitude analysis in neuromuscular diseases. Electromyogr. Clin. Neurophysiol., 36 (8), 463-8.

[9] Taku, O., Toshikazu, K., Yoshiki, O., Kookho, L., Yasuhiko, K., Shunzo, A., Fumihiko, N. (2002). Power spectrum analysis of compound muscle action potential in carpal tunnel syndrome patients. $J$. Orthop. Surg., 10 (1), 67-71.

[10] Yana, K., Mizuta, H., Kajiyama, R. (1995). Surface electromyogram recruitment analysis using higher order spectrum. In IEEE 17th Annual Conference on Engineering in Medicine and Biology Society, 20-23 Sept. 1995. IEEE, Vol. 2, 1345-1346.

[11] Hussain, M.S., Reaz, M.B.I., Yasin, F.M., Ibrahimy, M.I. (2009). Electromyography signal analysis using wavelet transform and higher order statistics to determine muscle contraction. Expert Systems, 26 (1), 35-48.

[12] Shahid, S., Walker, J., Lyons, G.M., Byrne, C.A., Nene, A.V. (2005). Application of higher order statistics techniques to EMG signals to characterize the motor unit action potential. IEEE Trans. Biomed. Eng. 52 (7), 1195-1209. 
[13] Reaz, M.B.I., Hussain, M.S., Yasin, F.M. (2006). Techniques of EMG signal analysis: Detection, processing, classification and applications. Biol. Proced. Online, 8 (1), 11-35.

[14] Kumar, D.K., Pah, N.D., Bradley, A. (2003). Wavelet analysis of surface electromyography to determine muscle fatigue. IEEE Trans. Neural Syst. Rehabil. Eng., 11 (4), 400-406.

[15] Walker, J. (1999). A Primer on Wavelets and Their Scientific Applications. Chapman \& Hall.

[16] Nikias, C.L., Petropulu, A.P. (1993). Higher-Spectral Analysis: A Nonlinear Signal Processing Framework. Prentice Hall.

[17] Hinich, M.J. (1982). Testing for gaussianity and linearity of a stationary time series. Journal of Time Series Analysis, 3 (3), 169-176.

[18] Fackrell, J.W.A. (1996). Bispectral Analysis of Speech Signals. PhD Thesis, University of Edinburgh.
[19] Nikias, C.L., Raghuveer, M.R. (1987). Bispectrum estimation: A digital signal processing framework. Proc. IEEE, 75 (7), 869-891.

[20] Elgar, S., Guza, R.T. (1988). Statistics of bicoherence. IEEE Trans. Acoust. Speech Signal Process., 36 (10), 1667-1668.

[21] Motion Lab Systems, Inc., http://www.emgsrus.com

[22] Hermens, H.J., Bruggen, T.V. (1988). Simulation of fatigue related changes in spectral parameters of surface EMG. In Engineering in Medicine and Biology Society: Proceedings of the Annual International Conference of the IEEE, 4-7 Nov. 1988. IEEE, Vol. 4, 1706-1707.

[23] Hagberg, M., Ericson, B.E. (1982). Myoelectric power spectrum dependence of muscular contraction level of elbow flexors. Eur. J. Appl. Physiol., 48 (2), 147-156.

[24] Moritani, T., Nagata, A., Muro, M. (1982). Electromyographic manifestations of muscular fatigue. Med. Sci. Sports Exerc., 14, 198-202. 